\title{
Core-shell Regeneration Magnetic Molecularly Imprinted Polymers-based SERS for Sibutramine Rapid Detection
}

Zhigang Liu, ${ }^{1}$ Yan Gao, ${ }^{1}$ Li Jin, ${ }^{2}$ Hua Jin, ${ }^{1} \mathrm{Na} X u,{ }^{3}$ XiaoYang Yu, ${ }^{2}$ Shihua Yu ${ }^{*, 2}$

${ }^{1}$ Centre of Analysis and Measurement, Jilin Institute of Chemical Technology, Jilin, 132022 China.

${ }^{2}$ College of Chemical \& Pharmaceutical Engineering, Jilin Institute of Chemical Technology, Jilin 132022 China.

${ }^{3}$ College of Materials Science and Engineering, Jilin Institute of Chemical Technology, Jilin 132022, China.

*(Author for correspondence: e-mail: ysh@jlict.edu.cn) 
Table S1. A comparison of methods for SIB detection.

\begin{tabular}{|c|c|c|c|}
\hline No. & Methods & Detection limit & Ref. \\
\hline \multirow{4}{*}{1} & & \multirow{4}{*}{$1.5 \mu \mathrm{g} / \mathrm{mL}$ (standard } & Int. J. Pharm. \\
\hline & HPLC & & Sci. Rev. Res. \\
\hline & & & 2014, 29(1), \\
\hline & & & $171-174$ \\
\hline \multirow{3}{*}{2} & & \multirow{4}{*}{$1.3 \mathrm{ng} / \mathrm{mL}$ (standard } & Lat. Am. J. \\
\hline & LC-ESI-MS & & Pharm. 2013, 32 \\
\hline & & & (8): 1164-1169 \\
\hline \multirow{3}{*}{3} & & & Biomed. Res. \\
\hline & UHPLC-MS & 一 & 2018, 29 (14): \\
\hline & & & $3006-3009$ \\
\hline \multirow{4}{*}{4} & & & Rapid Commun. \\
\hline & & $10 \mathrm{ng} / \mathrm{mL}$ & \\
\hline & GC-MS & $1 \cdot$ & Mass Spectrom. \\
\hline & & & 2007, 21: 79-88. \\
\hline \multirow{4}{*}{5} & & & J. Pharmaceut. \\
\hline & Ion mobility spectrometry & & \\
\hline & & $2 \mathrm{ng} / \mu \mathrm{L}$ (samples) & Biomed. 2011, \\
\hline & & & $54,469-474$ \\
\hline \multirow{3}{*}{6} & injection & & \\
\hline & & $2.48 \times 10^{-9}$ & J. Lumin. 2011, \\
\hline & (FI-ECL) & (standard solution) & $131,1515-1519$ \\
\hline 7 & Capillary electrophoresis & $\mu \mathrm{g} / \mathrm{mL}$ & Methods \\
\hline
\end{tabular}


(CE)

9 MMIPs-SERS (standard solution) $\quad \mathbf{2 0 1 6}, 8,621-626$.

$1.0 \times 10^{-9} \quad \mathrm{~mol} / \mathrm{L}$

This work

(standard solution)

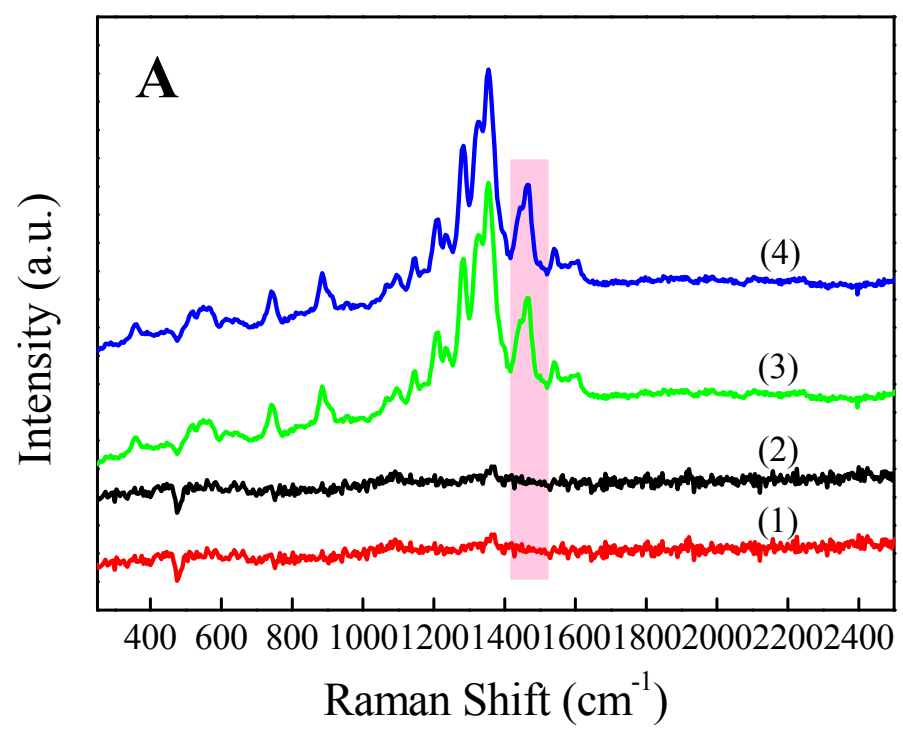

Figure S1. Raman spectra recorded on (1) a MIP-coated surface after the first regeneration, (2) a MIP-coated surface after the second regeneration, (3) a MIP-coated surface exposed to $1.0 \times 10^{-7} \mathrm{M} \mathrm{SIB}$, (4) a regenerated MIP-coated surface exposed to $1.0 \times 10^{-7} \mathrm{M}$ SIB.
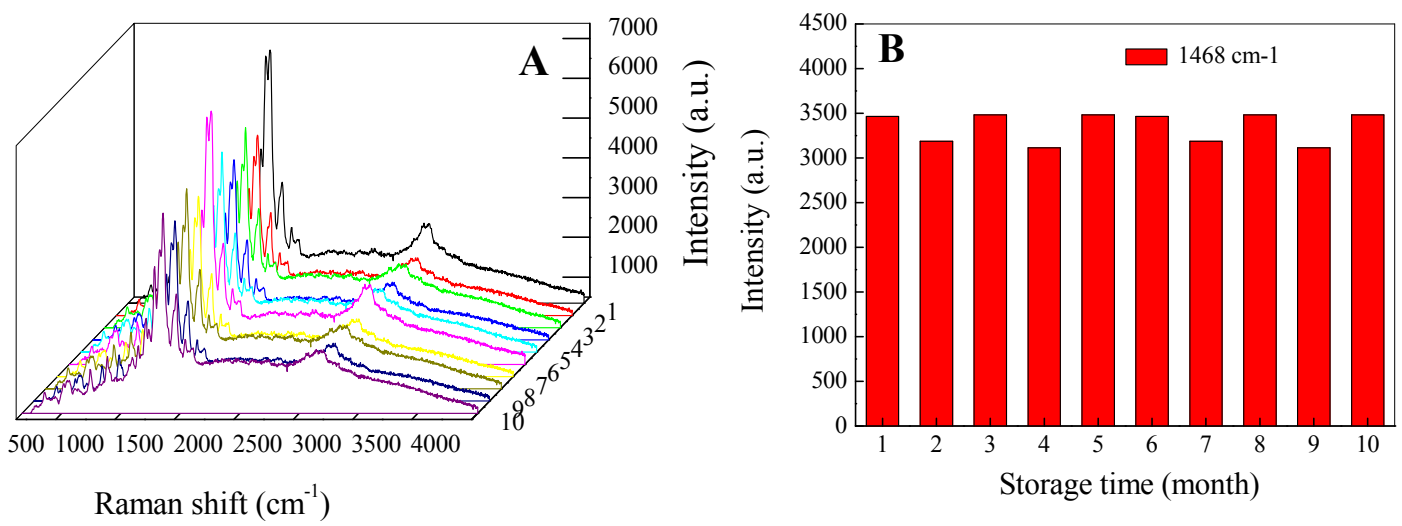
Figure S2. Reproducibility and stability of $\mathrm{Fe}_{3} \mathrm{O}_{4} @ \mathrm{Ag} @ \mathrm{MIPs}$ (A) Substrate-to-substrate Raman spectra for different storage times. (B) The corresponding Raman intensity variation at $1468 \mathrm{~cm}^{-1}$.
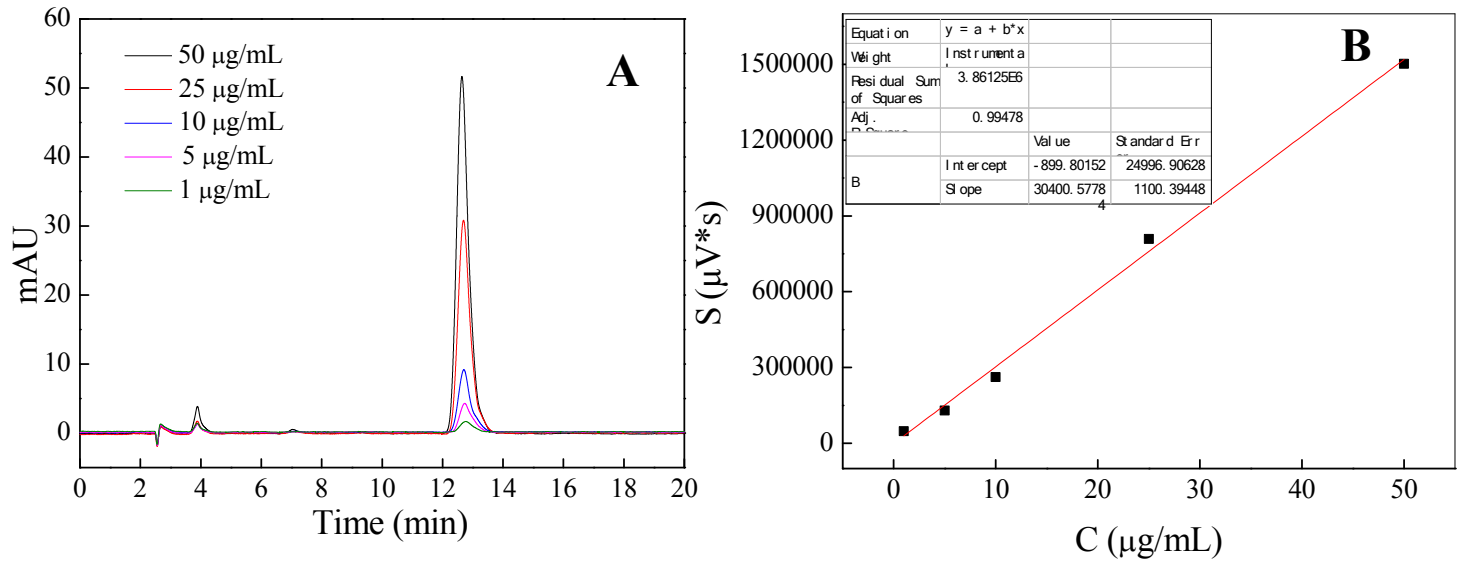

Figure S3. (A) Chromatograms of CAP at different concentrations; (B) Standard curves of CAP from the data of (A). 